\title{
Prostaglandins and their receptors in insect biology
}

\author{
David Stanley ${ }^{1}$ and Yonggyun Kim ${ }^{2}$ \\ ${ }^{1}$ Biological Control of Insects Research Laboratory, Agricultural Research Service, United States Department of Agriculture, Columbia, MO, USA \\ 2 Department of Bioresource Sciences, Andong National University, Andong, Republic of Korea
}

\section{Edited by:}

Joe Hull, USDA Agricultural Research

Service, USA

\section{Reviewed by:}

Qi Fang, Zhejiang University, China Qisheng Song, University of Missouri, USA

\section{*Correspondence:}

David Stanley, Biological Control of Insects Research Laboratory, Agricultural Research Service, United States Department of Agriculture, 1503 South Providence Road, Columbia, MO 65203, USA. e-mail: stanleyd@missouri.edu
We treat the biological significance of prostaglandins (PGs) and their known receptors in insect biology. PGs and related eicosanoids are oxygenated derivatives of arachidonic acid (AA) and two other $\mathrm{C} 20$ polyunsaturated fatty acids. PGs are mostly appreciated in the context of biomedicine, but a growing body of literature indicates the biological significance of these compounds extends throughout the animal kingdom, and possibly beyond. The actions of most PGs are mediated by specific receptors. Biomedical research has discovered a great deal of knowledge about PG receptors in mammals, including their structures, pharmacology, molecular biology and cellular locations. Studies of PG receptors in insects lag behind the biomedical background, however, recent results hold the promise of accelerated research in this area. A PG receptor has been identified in a class of lepidopteran hemocytes and experimentally linked to the release of prophenoloxidase. PGs act in several crucial areas of insect biology. In reproduction, a specific $\mathrm{PG}, \mathrm{PGE}_{2}$, releases oviposition behavior in most crickets and a few other insect species; PGs also mediate events in egg development in some species, which may represent all insects. PGs play major roles in modulating fluid secretion in Malpighian tubules, rectum and salivary glands, although, again, this has been studied in only a few insect species that may represent the Class. Insect immunity is a very complex defense system. PGs and other eicosanoids mediate a large number of immune reactions to infection and invasion. We conclude that research into PGs and their receptors in insects will lead to important advances in our understanding of insect biology.

Keywords: prostaglandins, insect physiology, receptors, immunity, reproduction

\section{INTRODUCTION}

Prostaglandins (PGs) and other eicosanoids are oxygenated derivatives of arachidonic acid (AA) and two other $\mathrm{C} 20$ polyunsaturated fatty acids. Corey et al. (1980) put forth the term eicosanoid, based on the Greek word for 20, "eikosi." Eicosanoid is a broad term for all biologically active AA metabolites. Three major groups of eicosanoids are recognized. The cyclooxygenase (COX) pathways lead to PGs; the lipoxygenase (LOX) pathways produce a myriad compounds, including the leukotrienes; and the cytochrome P-450 "epoxidase" pathways yield a group of epoxyeicosatrienoic acids. PGs and LOX products have been identified and exert biological actions in insect tissues. The epoxyeicosatrienoic acids act in mammalian tissues, but have not been reported for invertebrates. Figure 1 presents a broad outline of eicosanoid biosynthesis; eicosanoid chemical structures and biosynthetic pathways are treated in detail elsewhere (Stanley, 2000, 2005).

The roots of endocrinology go back to the 1850s, first with Arnold Berthold's castration/transplantation experiments on roosters (Benedum, 1999). He showed that castrating roosters led to atrophy of the comb and disinterest in hens. Also in the 1850s the French physiologist, Claude Bernard, discussed "internal secretions" that travel in blood circulation to distant target cells within the body. Eicosanoids are relative newcomers to biochemical signaling. The first known eicosanoids are dated to the 1930s, when von Euler (1936) reported that a substance(s) from the human prostate gland stimulated contractions in isolated uterine smooth muscle preparations. The contraction-stimulating substances had the characters of an acidic lipid and von Euler named them "prostaglandins" after their source. There was no real progress on PGs until Bergström and Samuelsson (Bergström et al., 1962) reported the chemical structures of PGE, $\mathrm{PGF}_{1}$ and $\mathrm{PGF}_{2}$ nearly 30 years later. Once the chemical structures of these PGs were revealed, it became clear immediately that AA is the immediate precursor for all PGs. Although originally interested in cholesterol reaction mechanisms, after the structural work on PGs, Bergström devoted his research to understanding AA metabolism, during which he discovered other eicosanoids, including endoperoxides, leukotrienes and thromboxanes. The pharmacologist John Vane shared the 1982 Nobel Prize for Medicine or Physiology with Bergström and Samuelsson for their pioneering work on PGs and other eicosanoids. Vane's contribution was his original demonstration that aspirin and other analgesics relieve discomforts associated with inflammation by inhibiting PG biosynthesis. Knowledge of the chemical structures and clinical significance of PGs and other eicosanoids launched a very large research enterprise in industry, academia and government. We now know that PGs and other eicosanoids are biosynthesized and exert important biological actions in virtually all mammalian tissues and body fluids. For a few examples, eicosanoids act in reproduction, immunity, smooth muscle contraction, ion transport and in mucosal protection. 


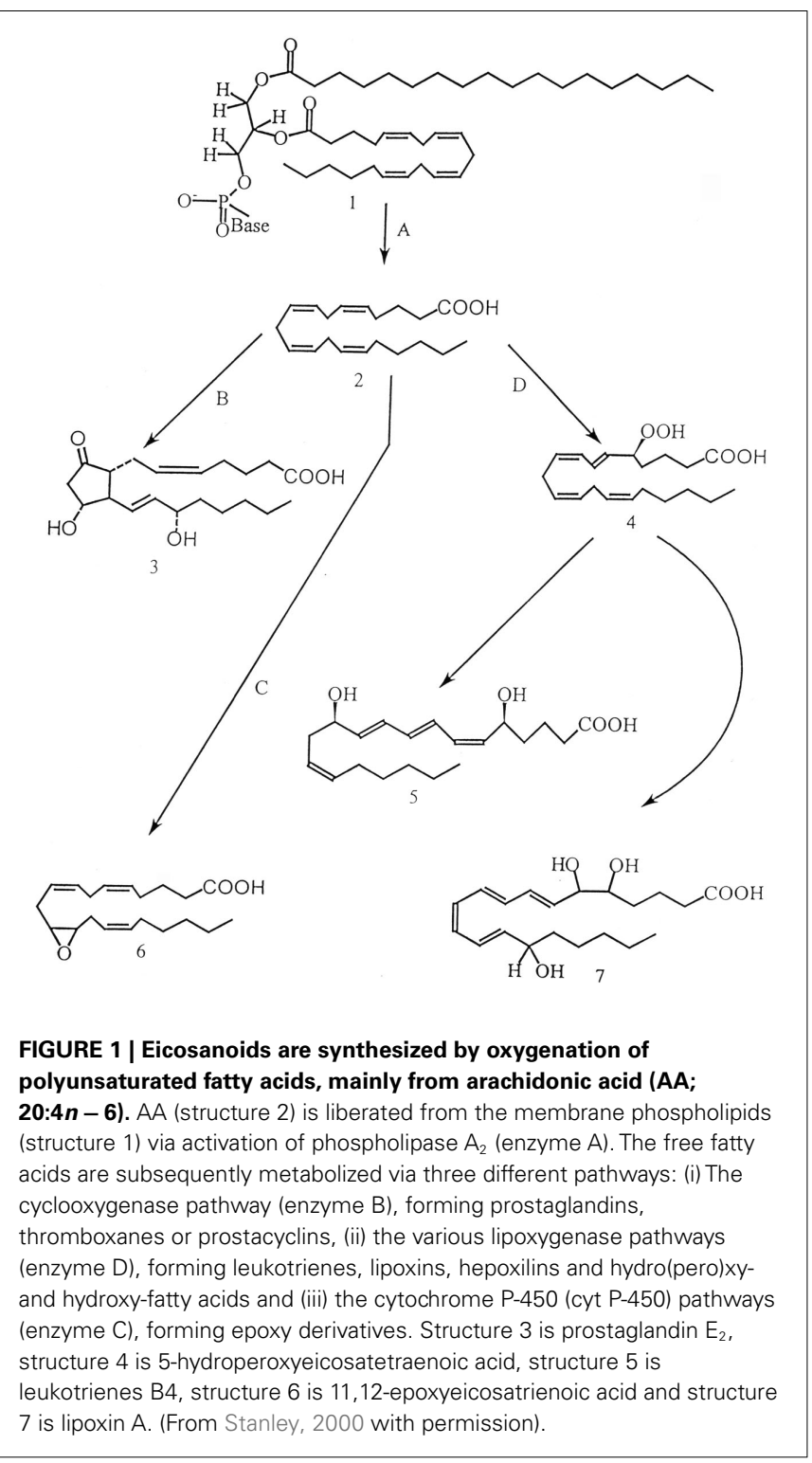

The discovery of eicosanoids in a marine invertebrate came from a broad research program on marine natural products. In their 15th paper in a series, Weinheimer and Spraggins (1969) reported on two PG derivatives in the gorgonian octocoral, Plexaura homomalla. As a side note, this discovery came before PGs were available for physiological research. While P. homomalla became the first commercial source of PGs for research purposes, there were no insights into the biological significance of PGs or any eicosanoids in invertebrates. Destephano and Brady (1977) provided the first suggestion that PGs act in insect biology with their report on PG biosynthesis in reproductive tissues of the house cricket, Acheta domesticus. They also showed that injecting $100 \mu \mathrm{g}$ of $\mathrm{PGE}_{2}$ into gravid virgin females led to release of egg-laying behavior. This was the first report of a biological role of PGs in insects.

In the context of this issue of Frontiers in Experimental Endocrinology, we focus on PG receptor-mediated events in insect physiology, including the release of egg-laying behavior in crickets, roles of PGs in insect egg development, a PG receptor in tick salivary glands and finally, a PG receptor-mediated action in insect immunity. The main points of the paper are that (1) in most cases, the PG receptors are postulated on the basis of specificity of PG actions, rather than studied directly; (2) PGs are crucial elements in insect physiology; (3) PG actions are receptor-mediated; and (4) research into insect PG receptors represents a very broad, newly illuminated frontier with profound implications in basic and applied biology.

\section{A BIOMEDICAL BACKGROUND ON PG RECEPTORS}

Drawing on the biomedical model developed in studies of mammalian physiology and pathophysiology, PGs and at least some LOX products interact with specific cell surface receptors, particularly G protein coupled receptors (GPCRs) of the rhodopsin family (Breyer et al., 2001). Figure 2 presents a model of one mechanism of the influence of PGs on cellular events. The genes encoding GPCRs account for relatively large proportions of animal genomes; GPCRs are organized into a variety of families (Vanden Broeck, 2001). GPCRs selectively couple with various intracellular $G$ proteins, each of which regulates the activities of specific cellular effector proteins, including adenylyl cyclase and others (Blenau and Baumann, 2001). Some mammalian PG receptors influence events related to homeostasis, such as ion transport physiology and others influence gene expression in target cells.

The detailed situation is still more complex. Whereas most PG receptors are thought to be located on cell surfaces, nuclear PG receptors also have been reported (Bhattacharya et al., 1999). Cell surface PG receptors also exist in various subtypes. PGs D, E, F, I, and Thromboxane A have specific receptors, each denoted by a letter associated with the PG and a P: DP, EP, FP, IP, and TP receptors. Still more complexity attends the EP receptors, which occur in subtypes, EP1, EP2, EP3, and EP4 and each of these interact with separate $G$ proteins. EP3 receptors are further subdivided into variants due to splice variations (Hatae et al., 2002). All this variation in PG receptors allows various PGs to exert multiple and even contradictory influences on cells. For example, EP3 variant EP3A, once activated, can stimulate increased intracellular cAMP concentrations, while EP3 variant EP3B lowers intracellular cAMP concentrations. Compared to the huge body of literature on PG receptors in the biomedical literature, there is only scant knowledge these receptors in invertebrates. Here we assemble the diverse information into a unified picture.

\section{PROSTAGLANDINS IN INSECT REPRODUCTION}

In decades of detailed research Werner Loher and his students developed the most complete picture of a cricket mating system, studying the Australian field cricket, Teleogryllus commodus. Loher and Edson (1973) showed that mating exerts profound influences on female crickets. They reported the apparently odd situation that successful mating, per se, does not attenuate female receptivity; instead, males tend to corral females within mating arenas after mating until they deposit their eggs. Males call and court females, and this synchronizes sexual readiness in both sexes. Males produce spermatophores and express calling behavior on a circadian rhythm basis (Loher, 1984). Virgin 


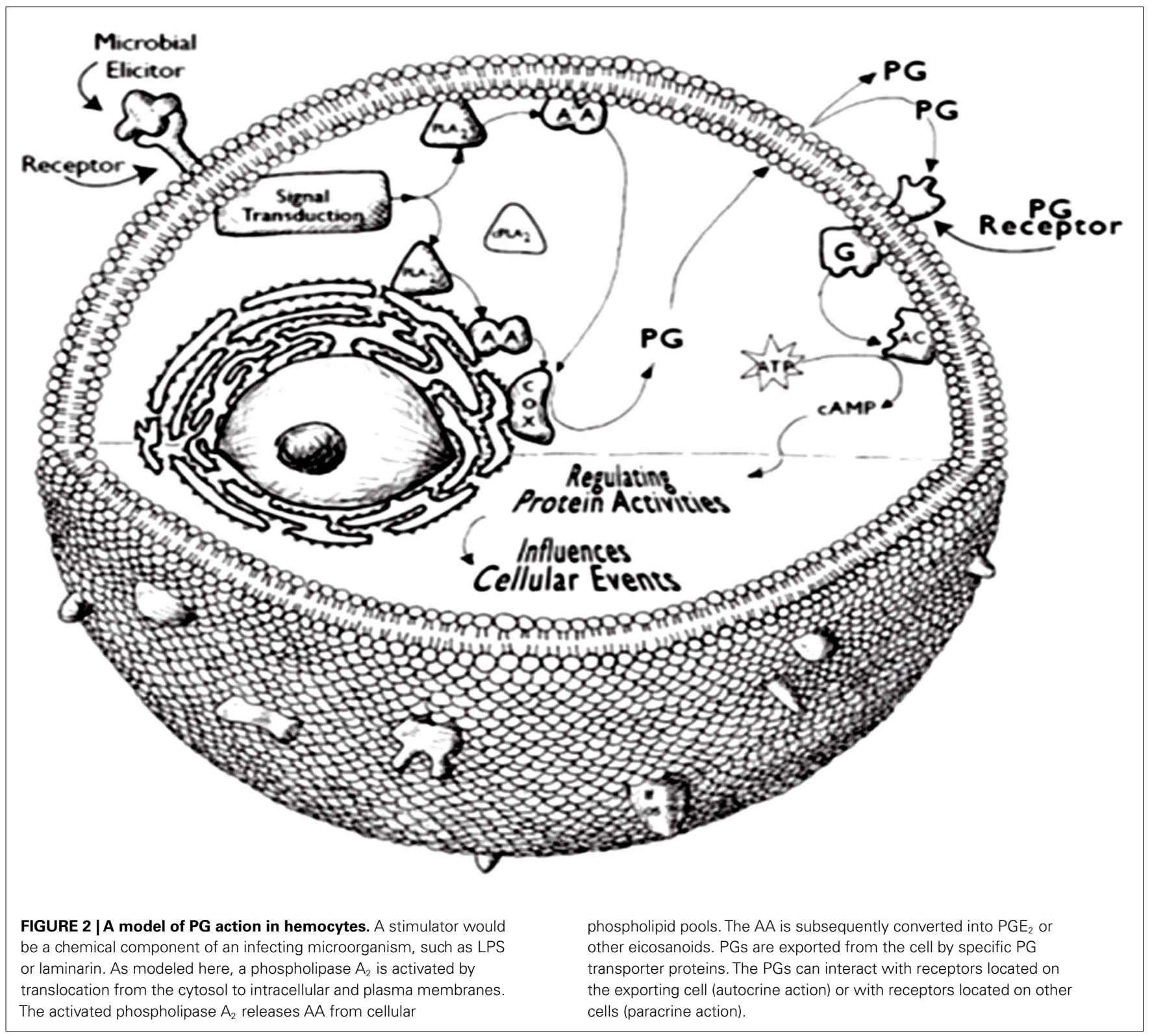

females express nightly locomotor behavior and are responsive to male calling songs, both behaviors also organized on circadian rhythms. Following successful mating (and not all copulations are successful because the long tubes of the spermatophores must find their way into the spermathecae), females stop their locomotor behaviors and lose interest in male calling songs (Loher, 1984). A third very important change in females is the onset of oviposition behavior that can last for hours and culminate in deposition of hundreds of fertile eggs, each individually deposited. Loher demonstrated that the physical stimulation of mating is not responsible for these profound changes in female behavior, because mating with castrated males does not produce the changes (Loher and Edson, 1973). He postulated that a chemical factor, produced in testes and sexually transferred to females, induced at least some of the behavioral changes in females.
In his first experiment to identify a chemical factor, Loher (1979) injected sexually mature virgins with $\mathrm{PGE}_{2}$ (and saline into control females), then recorded egg-laying each day for the following 13 days. For the first $24 \mathrm{~h}$ following treatment, Loher recorded an average of about 6 eggs/female in controls and about 76 eggs/female in PG-treated experimental females. Over the whole experiment experimental females deposited about 217 eggs/female/day, compared to about 11 by the control females. This work laid a foundation for a lengthy research program on the roles and actions of PG in releasing oviposition behavior in female crickets.

The program began with analytic biochemistry to determine quantities of $\mathrm{PGE}_{2}$ in spermathecae from virgin and mated females, using a high-performance liquid chromatography protocol, which at the time was sensitive to about $0.5 \mathrm{ng}$. They collected and analyzed pools of 100 spermathecae, from which they recorded 
about $500 \mathrm{pg} \mathrm{PGE} /$ spermatheca in glands from mated females and none in spermathecae from virgins. Recalling that PGs took their name from the prostate gland, mammalian semen is generally rich in PGs. On the basis of mammalian semen, Loher guessed that cricket seminal fluids, which are transferred to females in spermatophores, would contain a fair amount of PG. Similar chemical determinations showed that spermatophores contain about $20 \mathrm{pg} / \mathrm{spermatophore}$, far too little to account for the recorded $500 \mathrm{pg} / \mathrm{spermathecae.}$

The next line of work was designed to determine $\mathrm{PGE}_{2}$ biosynthesis in spermatophores and spermathecae. They used a fairly standard protocol in which the fluid contents of spermatophores and spermathecae were incubated in the presence of radioactive AA. After the incubations, the reactions were extracted and analyzed on thin-layer chromatography (TLC). This work showed that spermathecal preparations from virgin females did not produce PGs, while similar preparations from mated females and from spermatophores produced about 25-35 pmol $\mathrm{PGE}_{2} / \mathrm{h} /$ tissue and about $12-13 \mathrm{pmol} \mathrm{PGF} 2 \alpha / \mathrm{h} /$ tissue. Because the presence and biological significance of AA and other C20 polyunsaturated fatty acids was not appreciated at the time, the authors also recorded the presence of AA in spermathecae of virgin females at about $2 \%$ of total fatty acids. These findings became the basis of the "enzyme transfer model," which specified that an enzyme with PG biosynthetic activity was transferred from male to female along with the other components of spermathecal seminal fluid; once in the spermathecae of newly mated females, the enzyme converts $\mathrm{AA}$ into $\mathrm{PGE}_{2}$, which subsequently releases oviposition behavior (Loher et al., 1981). The model also prompted deeper study.

Stanley-Samuelson and Loher (1983) investigated the arrangement of AA in spermatophores and spermathecae. They found that spermathecae from mated females contained about 40fold more AA than spermathecae from virgin females $(0.008 \mathrm{mg}$ $\mathrm{AA} /$ spermatheca for virgins and about $0.34 \mathrm{mg} \mathrm{AA} / \mathrm{spermatheca}$ for mated females). The difference between virgin and mated females was roughly the amount of AA detected in spermatophores. Virtually all the AA in spermatophores was associated with phospholipids, with none in neutral lipids. Within phospholipids, AA made up about $4 \%$ of phosphatidylethanolamine fatty acids and about $25 \%$ of phosphatidylcholine fatty acids. This is a very unusual asymmetry in fatty acid arrangements, from which the authors inferred that AA is arranged in spermatophores for a special purpose.

All previous work had focused on the influence of a single PG, namely $\mathrm{PGE}_{2}$, on releasing egg-laying behavior. Of course, there are many PGs, which raises questions about the specificity of PG actions in releasing oviposition behavior. If the PG mode of action were similar to the mammalian background, specific PG receptors would limit the number of different PGs that release oviposition behavior. Stanley-Samuelson et al. (1986) investigated the relation between PG structures and egg-laying. They used groups of 15-day-old virgin females. Some were untreated as negative controls, some were mated with intact males as positive controls and experimental groups were injected with $100 \mu \mathrm{g}$ of a chemical. The outcomes of these experiments are tabulated in Stanley-Samuslson et al. (1986), where we see that the most effective treatments were the positive mating controls and treatments with 15 -keto-PGE 2 , a metabolite of $\mathrm{PGE}_{2}$. $\mathrm{PGE}_{2}$ treatments also released egg-laying behavior, but at about $57 \%$ of the influence of mating. The authors interpreted the results in terms of PG receptors, which at that time were completed unknown in insect biology. There seemed to be a clear specificity for PGs with the ring features of PGE, that is, 9-keto, 11-hydroxy substitutions. PGD is similar to PGE, with 9hydroxy, 11-keto substitutions, however, PGD treatments did not lead to deposition of substantial numbers of eggs. Hence, it would be postulated that PGs release egg-laying behavior via a specific receptor.

Oviposition behavior in T. commodus is a complex operation involving sense cells in the ovipositor used to assess the quality of an egg-laying substrate, followed by fertilization and inserting the ovipositor deep into the substrate. Eggs are moved into the substrate by oviduct musculature. As mentioned just above, males tend to guard their female partners until they have deposited their eggs, a behavior thought to reduce sperm competition because mated females remain receptive to other males even though they do not respond to calling songs. By analogy to the mammalian uterus, it was thought that PGs release oviposition behavior by stimulating contractions of the oviduct musculature. This idea was laid to rest by Loher (1984) and by Cook et al. (1984), who reported that PGs do not stimulate contractions of oviduct muscles in the cockroach, Leucophaea maderae. The T. commodus oviposition behavioral program is located in the terminal abdominal ganglion. The current untested hypothesis is that $\mathrm{PGE}_{2}$ releases the oviposition via interactions with a $\mathrm{PGE}_{2}$ receptor located in the terminal abdominal ganglion.

Prostaglandins act in releasing oviposition behavior in a few other insect species, including the 28 spotted ladybird, the rice brown planthopper and possibly the silk moth, all reviewed in Stanley (2000). Beyond this, PGs have been recorded in the female reproductive tracts of several insect species and mating leads to increases in PG titers (Stanley, 2000). Machado et al. (2007) were the first to report on a biological role for PGs within female reproductive tracts. They cultured ovarioles from the silk moth, Bombyx mori (Daizo strain), then determined the influence of PG biosynthesis inhibitors on ovarian development. B. mori ovarioles can be cultured in vitro and they can enter and complete choriogenesis autonomously. Machado et al. (2007) showed that treating cultured ovarioles with aspirin and other inhibitors of PG biosynthesis sharply reduced choriogenesis and the inhibitory influence could be reversed by adding $\mathrm{PGF}_{2 \alpha}$ to the cultures. They also used a commercial polyclonal antibody in western blots to record the presence of a COX protein, which increased as oogenesis moved toward choriogenesis. Thus, $\mathrm{PGF}_{2 \alpha}$ acts in ovarian development in B. mori and likely other insects. This is a specific PG action, which presumably takes place via a specific receptor.

The Machado research group has a long history of research on their model insect, the blood-sucking bug, Rhodnius prolixus. They reported on the roles of PGs and other eicosanoids in immune reactions of $R$. prolixus and the mosquito Anopheles albimanus, which we will discuss in the immunity section of this article (de Medeiros et al., 2009). They recently reported that fungal infection increased production of $\mathrm{PGE}_{2}$ in $R$. prolixus and that the increased $\mathrm{PGE}_{2}$ titers led to arrest of oogenesis in adult females. This work connects PG actions to the growing understanding of 
ecological immunity, which informs understanding that immune reactions to infection and invasion entail considerable fitness costs, including reduced reproductive capacity (Rolff and Siva-Jothy, 2003). The positive influence of $\mathrm{PGF}_{2 \alpha}$ on ovarian development in B. mori and the inhibitory effect of $\mathrm{PGE}_{2}$ on oogenesis in $R$. prolixus prompt the hypothesis that these two PGs exert opposite effects on ovarian development. The concept of two PGs exerting opposite effects within a single system is solidly based in the biomedical background, because each of the PGs has its individual receptor. Here we suggest insect ovarian tissues similarly express individual receptors allowing two PGs to express opposite effects. Recognizing the hypothesis suffers from recording the opposite effects in two insect species, it remains a testable idea.

\section{PROSTAGLANDINS IN ION TRANSPORT PHYSIOLOGY}

Prostaglandins influence ion transport physiology in invertebrates and in at least three insect ion transporting tissues, salivary gland, Malpighian tubules and rectum (Stanley, 2000). In the first suggestion that PGs act in insect biology, Dalton (1977a) reported that $\mathrm{PGE}_{1}$ did not alter basal fluid secretion rates in salivary glands isolated from the blowfly, Calliphora erythrocephala, but did attenuate the normal stimulating influence of serotonin on fluid secretion rates. Serotonin influences fluid secretion via interaction with a cell surface receptor that increases intracellular cAMP concentrations. The attenuating effect of $\mathrm{PGE}_{1}$ could be due to decreasing activity of adenylate cyclase, the enzyme responsible for cAMP production or by stimulating phosphodiesterase, the enzyme that metabolizes cAMP into an inactive form. Dalton (1977b) concluded that $\mathrm{PGE}_{1}$ does not influence phosphodiesterase, but slows adenylate cyclase. This work added PGs to the biochemicals known to influence ion transport in insects, but the work was not carried forward, probably because a theoretical basis for PG actions in insect biology had not yet been established.

Petzel and Stanley-Samuelson (1992) hypothesized that PGs modulate fluid secretion rates in Malpighian tubules in female mosquitoes, Aedes aegypti. They used the classical Ramsey assay in which isolated sets of five Malpighian tubules in $20 \mu \mathrm{l}$ saline drops were placed under light paraffin oil. Individual tubules were drawn into the oil. Fluid excreted by the tubules was visible in the oil and measured under a microscope. The experimental work showed that specific inhibitors of PG biosynthesis slowed the basal fluid secretion rates, while inhibitors of LOXs or epoxygenases did not influence fluid secretion. They concluded that PGs modulate basal fluid secretion in mosquito Malpighian tubules. In follow-up work, they used immunohistochemistry to localize $\mathrm{PGE}_{2}$ in principal, but not stellate cells, within Malpighian tubules and also recorded the presence of AA in phospholipids, but not neutral lipids, prepared from isolated Malpighian tubules (Petzel et al., 1993). Using Ramsay procedures modified for very small insects, Van Kerkhove et al. (1995) reported that inhibitors of PG biosynthesis, but not inhibitors of LOXs or epoxygenase, severely restricted basal fluid secretion in Malpighian tubule preparations from the forest ant, Formica polyctena. Again, the authors concluded that PGs modulate Malpighian tubule fluid secretion in insects.

The insect rectum makes up a major component of insect renal function, acting to transport water and selected metabolites from the rectal lumen back into hemolymph. The rectum of the locust, Locusta migratoria, is large enough to set up in an everted sac preparation to directly investigate regulation of rectal transport functions. Radallah et al. (1995) reported that treating everted rectal sacs with $\mathrm{AA}$ or with $\mathrm{PGE}_{2}$ led to dose-dependent increases in fluid reabsorption. The experimental dosages were well within a physiological range $\left(10^{-9}-10^{-6} \mathrm{M}\right)$ and similar treatments also led to increased intracellular $\mathrm{Ca}^{++}$concentrations and increased phospholipase $\mathrm{C}$ activity. This work allows the hypothesis that $\mathrm{PGE}_{2}$ acts via a specific receptor to stimulate the phospholipase C-driven signal pathway.

Salivary glands of the tick, Amblyomma americanum, and other tick species, produce copious amounts of PGs, which are injected into their hosts, along with salivary gland secretions which may also contain various pathogens. The PGs facilitate blood feeding by down-regulating host immune responses to the presence of the tick. The salivary glands also are responsible for renal functions during blood feeding on vertebrate hosts by injecting water and ions back into their hosts. The renal functions are regulated in part by $\mathrm{PGE}_{2}$, which acts via a specific receptor. Qian et al. (1997) used classical binding protocols to investigate a $\mathrm{PGE}_{2}$ receptor from salivary glands of the tick, A. americanum. The receptor meets all criteria of a specific $P G$ receptor. It is specific to $\mathrm{PGE}_{2}$, is reversible and is saturable. $\mathrm{PGE}_{2}$ acts through this receptor to mediate two physiological processes in tick salivary glands. $\mathrm{PGE}_{2}$ acts in salivary secretions and acts in receptor-mediated protein exocytosis (Qian et al., 1998). This work firmly establishes a PG receptor in tick renal function. Taken with all the work on the roles of PGs in fluid secretion, just described, we assert that PG-producing invertebrates also express GPCRs for the PGs. We turn, now, to insect immunity, another area of insect biology in which a specific PG receptor operates.

\section{PROSTAGLANDINS AND OTHER EICOSANOIDS ACT IN INSECT IMMUNITY}

Insects and other invertebrates have served as research model systems since the beginning of immunological research. For a couple of examples, Elie Metchnikoff, a Russian embryologist, placed splinters into the transparent bodies of bipinnaria stage starfish, and later observed phagocytes surrounding the splinter. Aside from the early work on vaccines, this is probably the first experiment in immunology. Another Russian, Serguei Metalnikov worked in the Pasteur Institute, where he conducted research on insect immunity in the 1920s. He is regarded as one of the founders of insect immunology.

Insects lack the antibody-based adaptive immune systems seen in vertebrates. Insect immunity is exclusively innate, that is, a naturally occurring, non-specific immunity that does not depend on previous infection experience. Nonetheless, insect immunity is a highly effective protection system. Insect immunology is studied from several perspectives. Direct studies of immune functions forms the basis of a large literature on the cellular and molecular mechanisms of immunity, and immune signaling, some of which has also guided biomedical research into mammalian immunity. The significance of research into insect immunity is underscored by recognition of Professor Jules Hoffmann, who led research into Drosophila immunity, with a share of the 2011 Nobel Prize 
in Physiology or Medicine "for their discoveries concerning the activation of innate immunity." Immune functions are biologically expensive and studies of ecological immunity are revealing physiological trade-offs, in which biologically expensive immune functions are traded off for other biological functions, including reproduction and migrations (Rolff and Siva-Jothy, 2003). For a single example, Schmid et al. (2008) reported that adult honeybees abandon cellular-based immunity in favor of prophenoloxidasebased immunity when they reach the foraging stage of their behavioral ontology. All this research highlights the breadth and depth of insect immunology, which is now beyond the capacity of individuals or even groups to comprehensively review. Let us begin with a brief glance of insect immune functions.

Insect integuments serve as exoskeletons and also as physical barriers to infectious agents. The gastrointestinal tract is a common entry to insect bodies, although it is not a completely open door for them. Insects express epithelial immunity in salivary glands (Abdelsadik and Roeder, 2010), guts (Cronin et al., 2009), Malpighian tubules (Davies and Dow, 2009), and tracheal epithelia (Wagner et al., 2008) of Drosophila. Epithelial immunity is a humoral response, based on induced expression of genes encoding anti-microbial peptides. After infectious agents get into hemolymph circulation, internal signaling coordinates and mediates humoral and cellular immune reactions. Humoral reactions, again, involve biosynthesis of anti-microbial peptides, the enzyme lysozyme and release of prophenoloxidase (PPO) from circulating enocytoids, a type of hemocyte. These peptides appear in the hemolymph of infected insects about $6-12 \mathrm{~h}$ post-infection (PI). Far more detailed descriptions of humoral immunity, including non-self surveillance and the signaling pathways that lead to anti-microbial peptide expression are available (Lemaitre and Hoffmann, 2007).

The hemolymph of most insects contains approximately 4$6 \times 10^{6}$ circulating hemocytes per $\mathrm{ml}$. The main immune effecter cells of Lepidoptera are plasmatocytes and granulocytes. These cells clear microbes from circulation by phagocytosis and a process called nodulation, a form of encapsulation. Nodulation begins with microaggregation of hemocytes with adhering microbes (Figure 3A), which grow into nodules. In the last phase of nodulation, plasmatocytes surround the nodule and activate a PPO system that melanizes nodules, which are finally attached to an internal organ or inner body wall. The darkened nodules remain in the body for the life of the insect and they are easily visible at $40 \times$ (Figure 3B). Nodulation is responsible for clearing the majority of infecting microbes from circulation (Dunn and Drake, 1983). Invaders too large for phagocytosis, such as parasitoid eggs, are encapsulated in layers of hemocytes within resistant insects. These also are melanized and attached to the inner body wall or an internal organ. The melanization process produces reactive oxygen forms that may directly kill the invaders. Numbers of circulating hemocytes decline sharply during cellular responses and are replaced by hematopoiesis that takes place in small hematopoietic organs typically located in abdomens, but also in the thorax of some species. Millions of hemocytes may be invested in responses to a single infection event, which emphasizes the biological costs of hemocytic immunity (Stanley, 2006).
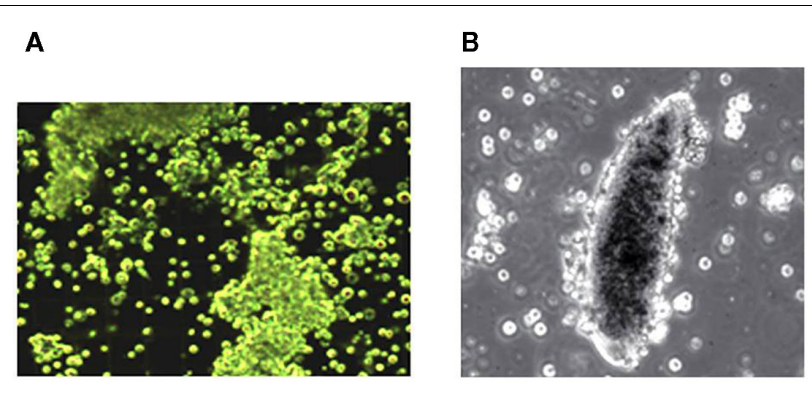

FIGURE 3 | (A) Microaggregation reactions to bacterial infection in larvae of the diamondback moth, Plutella xylostella. (B) Encapsulation in response to parasitoid egg $(200 \times)$. The cells in these photographs range from $10-12 \mu \mathrm{m}$. Nodulation is a form of encapsulation in which hemocytes surround a mature nodule rather than a parasitoid egg.

Stanley-Samuelson and his colleagues investigated the hypothesis that eicosanoids mediate insect hemocytic immunity. They reported that injecting tobacco hornworms, Manduca sexta, with pharmaceutical inhibitors of eicosanoid biosynthesis (the nonsteroidal anti-inflammatory drugs, NSAIDs) impaired the ability to clear injected bacteria from hemolymph circulation (StanleySamuelson et al., 1991). As all experiments were conducted within 2-4 h PI, it was suggested that eicosanoids mediated cellular, as opposed to humoral, immune reactions. A second paper reported that treating hornworms with inhibitors of eicosanoid biosynthesis impaired microaggregation and nodulation reactions to bacterial infection (Miller et al., 1994). These ground-breaking papers opened several lines of research. In one, several investigations of eicosanoid biochemistry documented the presence of eicosanoid precursor fatty acids in virtually all insects, recorded biosynthesis of PGs and other eicosanoids and demonstrated the presence of phospholipase $\mathrm{A}_{2}\left(\mathrm{PLA}_{2}\right)$ activity in immune and other insect tissues. These biochemical studies are beyond the scope of this article and specific citations more detailed reviews can be found elsewhere (Stanley, 2000, 2005, 2006; Stanley et al., 2009).

The biochemical work supported research into the influence of immune challenge on $\mathrm{PLA}_{2}$ activity and PG biosynthesis. Jurenka et al. (1999) reported that bacterial infection increased biosynthesis of $\mathrm{PGF}_{2 \alpha}$ in true armyworms, Pseudaletia unipuncta, at $30 \mathrm{~min}$ post-infection. This work bolstered the idea that PGs and other eicosanoids are key mediators of insect cellular immunity. Later several authors reported that infection stimulated increased $\mathrm{PLA}_{2}$ activity in tobacco hornworms (Tunaz et al., 2003), in whole flies, Sarcophaga peregrine (Yajima et al., 2003), in beet armyworms, Spodoptera exigua (Shrestha and Kim, 2007), and in the red flour beetle, Tribolium castaneum (Shrestha and Kim, 2010). Together, these publications allow a generalization that $\mathrm{PLA}_{2}$ acts in insect cellular immunity.

In the context of PG receptors, we now consider specific cellular actions mediated by eicosanoids. In the early work, Miller et al. (1994) showed that eicosanoids mediate microaggregation and nodulation reactions to bacterial infection. Mandato et al. (1997) enhanced the list of eicosanoid-mediated actions, with their report that eicosanoids act in PPO activation, phagocytosis and cell spreading in larval Galleria mellonella. The influence 
of eicosanoids on PPO activation is quite interesting because eicosanoids do not activate PPO in other species, such as a locust, L. migratoria (Goldsworthy et al., 2003). Figueiredo et al. (2008) also showed that inhibiting $\mathrm{PLA}_{2}$ substantially reduced phagocytosis of the protozoan parasite, Trypanosoma rangeli, by $R$. prolixus hemocytes and that the inhibition was reversed by treating $R$. prolixus with AA. They concluded that eicosanoids mediate phagocytosis in Rhodnius and that $T$. rangeli somehow inhibited eicosanoid biosynthesis. Carton et al. (2002) reported that the $\mathrm{PLA}_{2}$-inhibiting glucocorticoid, dexamethasone, inhibited encapsulation of parasitoid eggs in a resistant line of D. melanogaster. Similarly, Miller (2005) confirmed eicosanoid actions in M. sexta plasmatocyte spreading. Hemocyte adhesion is crucial in clearing infecting bacteria from circulation. Marin et al. (2005) reported that AA increased adhesion of G. mellonella granulocytes, but not plasmatocytes, to a glass surface. More recently, Merchant et al. (2008) reported that eicosanoids influence tobacco hornworm hemocyte migration in Boyden chambers. This may be a specific PG function because a LOX inhibitor, esculetin, did not influence hemocyte migration. Finally, Shrestha and Kim (2008) discovered that eicosanoids mediate release of PPO from enocytoids by inducing cell lysis in S. exigua. Hence, we have a list of specific eicosanoid actions beginning with microaggregation, encapsulation and nodulation as a form of encapsulation. These are large-scale, visible actions, each involving a large, but unknown number of discrete cell steps. For example, phagocytosis is a very complex action, which in Drosophila S2 cells involves at least 184 genes (Stroschein-Stevenson et al. (2006). Cell spreading and migration are similarly complex, although we do not have a direct comparator with respect to numbers of genes. We conclude that eicosanoids are crucial to the mediation and coordination of insect cellular immunity, but they exert their actions in concert with other biochemical signal systems, including biogenic amines and insect cytokines. Recent work suggests how eicosanoids interacts with other signal systems.

There are reports of specificity also in the broad groups of eicosanoids acting in immune functions. Lord et al. (2002) reported that eicosanoids, specifically LOXs products, but not by PGs, act in cellular immunity to the insect fungal pathogen, Beauveria bassiana. Similarly, PGs, but not LOXs products, appear to mediate microaggregation reactions to bacterial infection in isolated hemocyte preparations (Phelps et al., 2003). These reports also point to specific eicosanoid receptors, although the operational receptors have not been identified.

Baines et al. (1992) reported that two biogenic amines, octopamine (OA) and 5-hydroxy-tryptamine (5-HT), mediate phagocytosis and nodulation reactions to bacterial infection in the cockroach, Periplaneta americana, and subsequently showed that 5-HT acts through a GPCR that influences adenylate cyclase (Baines and Downer, 1992). Kim et al. (2009) were the first to investigate the intracellular interactions between eicosanoids and the biogenic amines in insect immunity. They showed that OA and 5-HT independently mediated phagocytosis and nodulation reactions in larvae of the lepidopteran, S. exigua. Two monoamine receptor antagonists, phentolamine and ketanserin, inhibited phagocytosis and nodulation and treating experimental larvae with the eicosanoid precursor fatty acid, AA, reversed the inhibitory influence of the receptor antagonists. Similarly, the mediating influence of OA and 5-HT on phagocytosis and nodulation was inhibited in larvae treated with dexamethasone, a $\mathrm{PLA}_{2}$ inhibitor that blocks all eicosanoid biosynthesis. The authors concluded that the immune-mediating influence of biogenic amines is expressed through stimulation of eicosanoid biosynthesis. This work demonstrated a solid intracellular connection between the actions of biogenic amines and eicosanoids in two cellular immune functions, phagocytosis and nodulation. This example of crosstalk between biogenic amine and eicosanoid signaling probably represents a common feature of signaling in insect immunity.

Plasmatocyte-spreading peptide (PSP) is an insect cytokine, first identified in the soybean looper, Pseudoplusia includens (Clark et al., 1997). Pro-PSP is activated by cleavage into a 23-residue PSP that mediates plasmatocytes spreading (PS; Clark et al., 1998). PSP acts through an approximately $190 \mathrm{kDa}$ receptor to induce PS (Clark et al., 2004). PS is a crucial action in insect innate immunity. The process of nodule formation following microbial infection is completed by plasmatocytes which spread over mature nodules, then activate a PPO to form a melanized, dark layer that completely encapsulates nodules. Plasmatocyte spreading also acts in responses to wounding by spreading over wounds to participate in hemolymph clotting. PSP likely occurs in all insects that express cellular immune reactions because the $P$. includens PSP also acts in other lepidopterans, including S. exigua (Kim et al., 2008).

Srikanth et al. (2011) investigated the possible cross-talk between PSP and eicosanoid signaling in S. exigua. They showed that both PSP and $\mathrm{PGE}_{2}$ independently mediate hemocyte spreading, that pharmaceutical inhibitors of $\mathrm{PLA}_{2}$ inhibit hemocyte spreading in a dose-dependent manner and the same inhibitors inhibit PSP-stimulated hemocyte spreading, also in a dosedependent manner. After cloning and sequencing the $S$. exigua gene encoding PSP, they showed that dsRNA designed to silence the gene effectively impaired cell spreading. The impairing influence of the dsRNA was rescued, in separate experiments, by treating dsRNA-injected larvae with AA and with PSP. The authors proposed a model (Figure 4) depicting the cross-talk between PSP and eicosanoid signaling. Infection stimulates production of activated PSP, which interacts with hemocytes through the PSP receptor. The PSP-receptor interaction activates a $\mathrm{PLA}_{2}$, which in turn stimulates $\mathrm{PGE}_{2}$ biosynthesis. The PSP-stimulated $\mathrm{PGE}_{2}$ biosynthesis leads to plasmatocytes spreading. This is another example of the cross-talk between eicosanoid and other signaling systems. This cross-talk between PSP and biogenic amine signaling establishes eicosanoids as an integral element of immune signaling in insect biology. The eicosanoid actions discussed in this section operate on the assumption of specific receptors for PGs and possibly other eicosanoids. Let us move on to the recent appreciation of a specific $\mathrm{PG}$ receptor operating in an S. exigua immune response.

Phenoloxidase (PO) is responsible for the melanization reactions to infection in insect and other invertebrates. These reactions are an enzyme-based element of humoral immune responses to infection, invasion and wounding. POs also act in other areas of insect biology, including tanning during post-embryonic development. Recent work on honeybee immunity emphasizes the significance of PO-based immunity, showing that adult honeybees abandon hemocytic immunity as they enter the foraging phase 


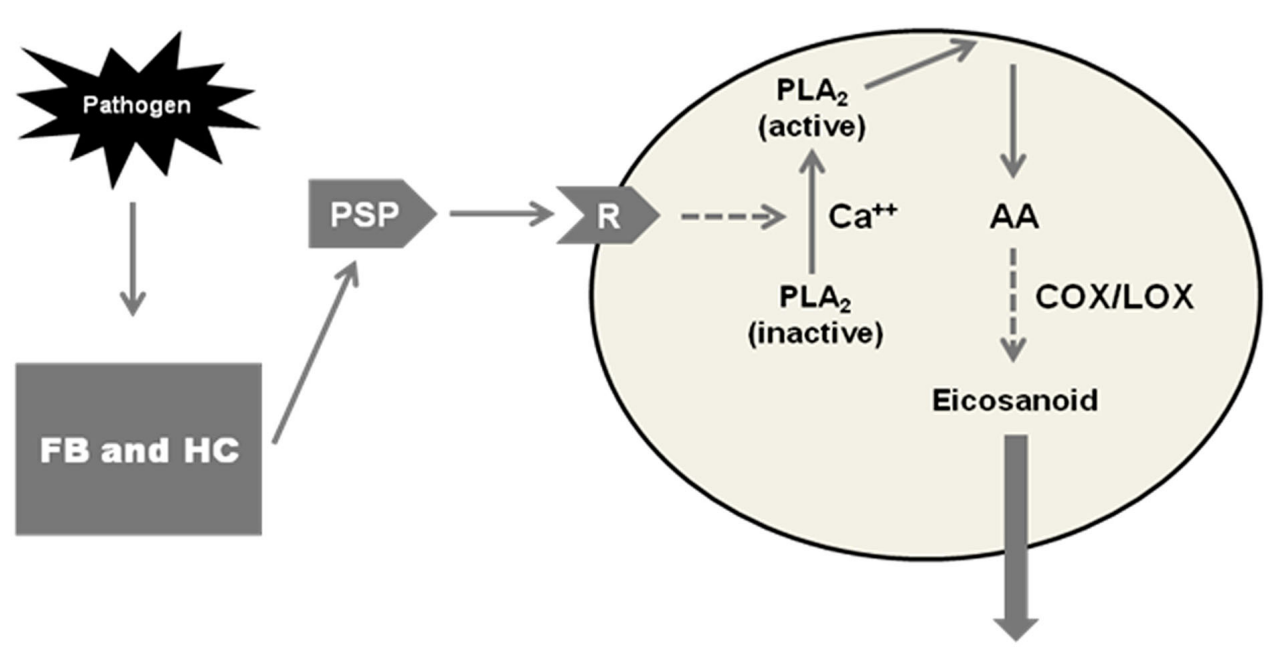

Hemocyte-spreading

FIGURE 4 | A model of a cross-talk between plasmatocyte-spreading peptide (PSP) and eicosanoids in mediating hemocyte-spreading behavior in Spodoptera exigua. In response to pathogens, fat body (FB), and hemocytes $(\mathrm{HC})$ release PSP, which then binds to a hemocyte receptor
(R). The binding to the PSP-receptor induces activation of phospholipase $A_{2}$ $\left(P L A_{2}\right)$, which hydrolyzes arachidonic acid (AA) from phospholipid substrates. $A A$ is then oxygenated by COX or LOX enzymes to form PGs, and possibly other eicosanoids, that mediate hemocyte-spreading behavior. of behavioral ontogeny; their PO-based immunity remains intact and even increases during foraging phase (Schmid et al., 2008). Inactive PPO is activated into PO by a series of serine proteases. In S. exigua, and likely most lepidopterans, PPO is produced and stored in specialized hemocytes, the enocytoids. PPO is released from enocytoids into hemolymph circulation by lysis and the lysis action is mediated by eicosanoids (Shrestha and Kim, 2008).

Shrestha et al. (2011) went on to show that a specific eicosanoid, $\mathrm{PGE}_{2}$, is responsible for enocytoid lysis. $\mathrm{PGE}_{2}$ acts through a GPCR, which they cloned and denoted Se-hcPGGPCR1. The receptor is expressed in all life stages, although rather faintly in eggs. Within larvae, it is expressed in hemocytes and gut, but not fat body or epidermis. Fluorescence in situ hybridization showed that the receptor is expressed in enocytoids, but not the other hemocyte types, including the more abundant plasmatocytes and granulocytes. The receptor is constitutively expressed in low levels, which are increased following infection. $\mathrm{PGE}_{2}$ also mobilizes intracellular $\mathrm{Ca}^{++}$during lysis (Shrestha and Kim, 2009). Shrestha et al. (2011) used a Ca ${ }^{++}$mobilization assay to establish a functional link between $\mathrm{PGE}_{2}$ and Se-hcPGGPCR1. They used hemocytes prepared from S. exigua larvae that were untreated (controls) or injected with dsRNA specific to Se-hcPGGPCR1. PGE 2 treatments increased intracellular $\mathrm{Ca}^{++}$concentrations in a dose-dependent manner over the physiological range of $10^{-12}-10^{-4} \mathrm{M}$ in untreated control hemocyte preparations and in dsRNA treated preparations. $\mathrm{Ca}^{++}$mobilization was significantly reduced in the experimental preparations in which the gene encoding hcPGGPCR1 was silenced.

Downstream signaling of $\mathrm{PGE}_{2}$ receptor binding was analyzed in enocytoid cell lysis (Shrestha and Kim, 2009). U-73122 (a specific inhibitor of phospholipase C) inhibited enocytoid lysis of
S. exigua significantly after bacterial infection. EGTA (a calcium chelator) treatment inhibited the cell lysis. Thus the enocytoid lysis depends on calcium signaling in response to $\mathrm{PGE}_{2}$. Two PKC inhibitors (staurosporine and calphostin C) significantly inhibited the enocytoid lysis. Enocytoid lysis was likely induced by $\mathrm{Na}^{+}$entry and subsequent osmotic shock because juvenile hormone analog, pyriproxyfen, which activates $\mathrm{Na}^{+}-\mathrm{K}^{+}$ATPase and induces subsequent cell shrinkage, antagonized the effect of eicosanoid on cell lysis. Furthermore, ouabain (a specific $\mathrm{Na}^{+}$pump inhibitor) significantly inhibited enocytoid lysis. These results suggest that eicosanoids mediate enocytoid lysis by activating the intracellular PKC pathway (Figure 5).

The specificity of PG actions in reproduction, fluid secretion and in enocytoid lysis supports the view that PG actions in insect biology are mediated via specific receptors. PGs express two general actions in cells. In one, they modulate homeostatic functions, such as basal fluid secretion rates, ovarian development and immune reactions. In another, PGs also influence expression of specific genes and proteins. Stanley et al. (2008) reported that challenging a cell line established from Helicoverpa zea (HzAM1 cells) with $\mathrm{PGA}_{1}$ and $\mathrm{PGE}_{1}$ led to changes in protein expression. The affected proteins included proteins in lipid metabolism, signal transduction, protection, cell functions and metabolism, however, each of the two PGs exerted different influences on protein expression. The specificity of the PG effects on protein expression also supports the view that PGs act through specific receptors.

\section{SOME MICROBES CRIPPLE INSECT IMMUNITY VIA INHIBITION OF PLA}

We close this article by looking at PG and other eicosanoid signaling from the perspective of some microbes. The point here is the 


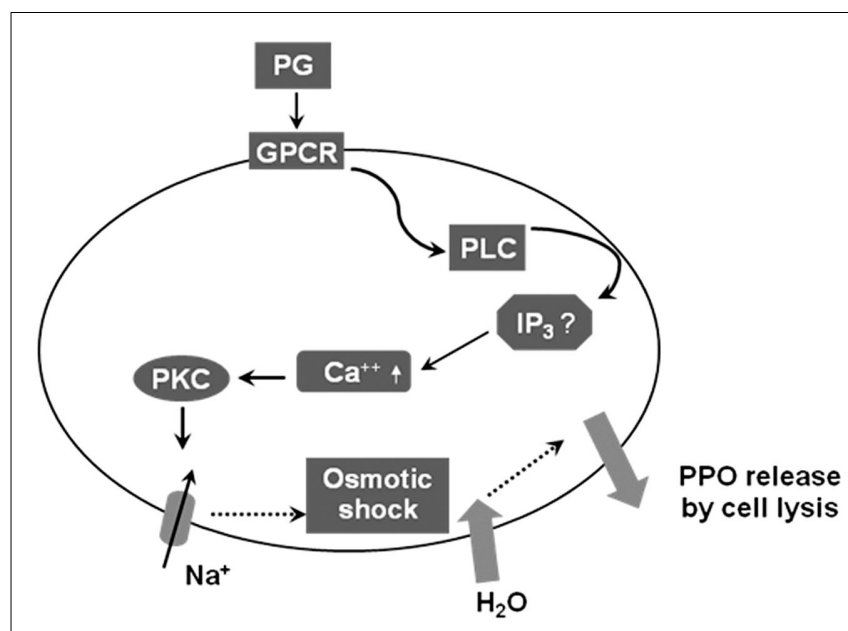

FIGURE 5 | A working hypothesis of the intracellular signal pathway of enocytoid lysis in response to PGs in Spodoptera exigua. Following bacterial challenge, PGs are produced and bind to membrane receptors ("GPCR") on enocytoids, which activates phospholipase C (PLC) and releases inositol trisphosphate $\left(\mathrm{IP}_{3}\right)$. The secondary messenger then up-regulates intracellular calcium level and activates protein kinase $C$ (PKC). The activated PKC may induce opening of $\mathrm{Na}^{+}$channels and increase osmotic pressure, which allows influx of water, responsible releasing prophenoloxidase (PPO) by lyzing the enocytoids.

microbes produce and secrete compounds specifically tailored to the active sites of some PLA 2 s. As described earlier, the first step in eicosanoid biosynthesis is a $\mathrm{PLA}_{2}$ step that hydrolyzes AA from cellular phospholipid pools.

The entomopathic nematode, Steinernema carpocapsae, is a mutualistic symbiont with the bacterium, Xenorhabdus nematophila, a member of the family Enterobacteriaceae. After entering an insect body, free-living, non-feeding juvenile nematodes void $X$. nematophila into the hemolymph. The bacteria rapidly proliferate and kill the insect host. The freshly killed insect body serves the mutualistic nematode partner in two ways. First, the insect cadaver provides the nematode with an appropriate microhabitat to complete development and reproduce, supplying nutrients and restricting growth of other microbes. Second, $X$. nematophila protects the nematode from hemocytic encapsulation by impairing the host immune reactions to the foreign nematode. In their research into the beet armyworm, $S$. exigua, Park and Kim (2000) first suggested that the bacterium impairs host immunity by inhibiting eicosanoid biosynthesis. Outcomes of their experiments documented that treating the nematode-challenged host larvae with AA reduced mortality by about $40 \%$. Deeper research revealed that X. nematophila produces and secretes heat-labile factors that directly inhibit secretory $\mathrm{PLA}_{2}\left(\mathrm{sPLA}_{2}\right)$ in the host. Moving beyond $S$. exigua, Park et al. (2003) reported that $X$. nematophilus similarly suppresses cellular immune reactions to infection by blocking eicosanoid biosynthesis in M. sexta. The bacterial inhibitory factor(s) occurred in the organic fraction of the bacterial culture medium and, as seen in the first experiment of Park and Kim (2000), the influence of the factor on cellular immunity was reversed by treating experimental hornworms with AA. In direct experiments with isolated $\mathrm{PLLA}_{2} \mathrm{~s}$, the authors demonstrated the bacterial factors are potent $s \mathrm{PLA}_{2}$ inhibitors: they inhibit $\mathrm{PLA}_{2}$ s from insect, prokaryotic and vertebrate sources (Park et al., 2004). The immunosuppressive action of the bacterium goes beyond Lepidoptera, to at least one cricket species, Gryllus firmus (Park and Stanley, 2006). Kim et al. (2005) showed that a related bacterium, Photorhabdus temperata (also a mutualistic partner of nematodes) similarly inhibits $\mathrm{PLA}_{2}$ and nodulation in its host, S. exigua. The authors suggested that bacteria in the genera Xenorhabdus and Photorhabdus generally share the ability to inhibit $\mathrm{PLA}_{2}$ in their hosts. The Enterobacteriaceae is a fairly large family of bacteria and it is not unreasonable to suppose other bacterial species in this family also impair insect host immunity by inhibition $\mathrm{sPLA}_{2} \mathrm{~s}$.

The strategy of impairing host immunity via compromising eicosanoid biosynthesis is not limited to bacteria. Garcia et al. (2004) described the results of a subtle experiment in this area. They first fed fifth-instar blood-sucking bugs, $R$. prolixus, on blood containing juveniles of the protozoan $T$. rangeli. T. rangeli is nonpathogenic protozoan parasite of $R$. prolixus. A few days later, they injected additional $T$. rangeli into the insects. This double treatment resulted in reduced hemocyte phagocytosis and increased host mortality. These effects were reversed in experiments in which protozoans were co-injected with AA (at $10 \mu \mathrm{g} /$ insect). The authors inferred that oral infection with $T$. rangeli inhibits the release of AA for eicosanoid biosynthesis. They also found that adding the glucocorticoid, dexamethasone, (which inhibits $\mathrm{PLA}_{2}$ in an indirect manner) to the blood meals of $R$. prolixus inhibited phagocytosis. The inhibition was reversed by treating experimental $R$. prolixus larvae with AA or, separately, with platelet activating factor, a specialized phospholipid (Figueiredo et al., 2008). They also showed that T. rangeli cells inhibit phagocytosis and the inhibition was reversed by AA treatments. They used a fluorometric assay to determine that $T$. rangeli cells inhibit $\mathrm{PLA}_{2}$ activity in $R$. prolixus hemocytes. This work shows that the protozoan parasite suppresses a host immune function, phagocytosis, by targeting the active site of $\mathrm{PLA}_{2}$ s that act in eicosanoid biosynthesis.

These findings support a broader generalization. Infectious organisms have evolved a very wide range of strategies to avoid or suppress insect immunity, most of which are outside the scope of this paper. These many strategies emerged from the tremendous selection forces on invaders. The fact that some of the invaders operate by inhibiting a key enzyme in eicosanoid signaling is a convincing argument that eicosanoids are crucial mediators of insect immunity.

\section{ACKNOWLEDGMENTS}

Thanks to Dr. Joe Hull, USDA/ARS, for the invitation to write this article. Mention of trade names or commercial products in this article is solely for the purpose of providing specific information and does not imply recommendation or endorsement by the U.S. Department of Agriculture. All programs and services of the U.S. Department of Agriculture are offered on a non-discriminatory basis without regard to race, color, national origin, religion, sex, age, marital status, or handicap. 


\section{REFERENCES}

Abdelsadik, A., and Roeder, T. (2010). Chronic activation of the epithelial immune system of the fruit fly's salivary glands has a negative effect on organismal growth and induces a peculiar set of target genes. BMC Genomics 11, 265. doi:10.1186/1471-2164-11-265

Baines, D., DeSantis, T., and Downer, R. G. H. (1992). Octopamine and 5-hydroxytryptamine enhance the phagocytic and nodule formation activities of cockroach (Periplaneta americana) hemocytes. J. Insect Physiol. 41, 905-914.

Baines, D., and Downer, R. G. H. (1992). 5-Hydroxytryptaminesensitive adenylate cyclase affects phagocytosis in cockroach hemocytes. Arch. Insect Biochem. Physiol. 21, 303-316.

Benedum, J. (1999). The early history of endocrine cell transplantation. $J$. Mol. Med. 77, 30-35.

Bergström, S., Ryhage, R., Samuelsson, B., and Sjovall, J. (1962). The structure of prostaglandin E, F1 and F2. Acta Chem. Scand. 16, 501-502.

Bhattacharya, M., Varma, D. R., and Chemtob, S. (1999). Nuclear prostaglandin receptors. Gene Ther. Mol. Biol. 4, 323-338.

Blenau, W., and Baumann, A. (2001). Molecular and pharmacological properties of insect biogenic amine receptors: lessons from Drosophila melanogaster and Apis mellifera. Arch. Insect Biochem. Physiol. 48, 13-38.

Breyer, R. M., Bagdassarian, C. K., Myers, S. A., and Breyer, M. D. (2001). Prostanoid receptors: subtypes and signaling. Annu. Rev. Pharmacol. Toxicol. 41, 661-690.

Carton, Y., Frey, F., Stanley, D. W., Vass, E., and Nappi, A. J. (2002). Dexamethasone inhibition of the cellular immune response of Drosophila melanogaster against a parasitoid. J. Parasitol. 288, 405-407.

Clark, K. D., Garczynski, S. F., Arora, A., Crim, J. W., and Strand, M. R. (2004). Specific residues in plasmatocyte-spreading peptide are required for receptor binding and functional antagonism of insect human cells. J. Biol. Chem. 279, 33246-33252.

Clark, K. D., Pech, L. L., and Strand, M. R. (1997). Isolation and identification of a plasmatocyte-spreading peptide from the hemolymph of the lepidopteran insect Pseudoplusia includens. J. Biol. Chem. 272, 23440-23447.
Clark, K. D., Witherell, A., and Strand, M. R. (1998). Plasmatocyte spreading peptide is encoded by an mRNA differentially expressed in tissues of the moth Pseudoplusia includens. Biochem. Biophys. Res. Commun. 250, 479-485.

Cook, B. J., Holman, G. M., and Meola, S. (1984). The oviduct musculature of the cockroach Leucophaea maderae and its response to various neurotransmitters and hormones. Arch. Insect Biochem. Physiol. 1, 167-178.

Corey, E. J., Albright, J. O., Barton, A. E., and Hashimoto, S. (1980). Chemical and enzymic synthesis of 5HPETE, a key biological precursor of slow-reacting substance of anaphylaxis (SRS) and 5-HETE. J. Am. Chem. Soc. 102, 1435-1436.

Cronin, S. J. F., Nehme, N. T., Limmer, S., Liegeois, S., Pospisilik, J. A., Schramek, D., Leibbrandt, A., de Matos Simoes, R., Gruber, S., Puc, U., Ebersberger, I., Zoranovic, T., Neely, G. G., von Haeseler, A., Ferrandon, D., and Penninger, J. M. (2009). Genome-wide RNAi screen identifies genes involved in intestinal pathogenic bacterial infection. Science 325, 340-343.

Dalton, T. (1977a). Threshold and receptor reserve in the action of 5hydroxytryptamine on the salivary glands of Calliphora erythrocephala. J. Insect Physiol. 23, 625-631.

Dalton, T. (1977b). The effect of prostaglandin E1 on cyclic AMP production in the salivary glands of Calliphora erythrocephala. Experientia 33/10, 1329-1330.

Davies, S.-A., and Dow, J. A. T. (2009). Modulation of epithelial innate immunity by autocrine production of nitric oxide. Gen. Comp. Endocrinol. 162, 113-121.

de Medeiros, M. N., Belmonte, R., Soares, B. C. C., de Medeiros, L. N., Canetti, C., Freire-de-Lima, C. G., Maya-Monteiro, C. M., Bozza, P. T., Almeida, I. C., Masuda, H., Kurtenbach, E., and Machado, E. A. (2009). Arrest of oogenesis in the bug Rhodnius prolixus challenged with the fungus Aspergillus niger is mediated by immune response-derived PGE2. J. Insect Physiol. 55, 151-158.

Destephano, D. B., and Brady, U. E. (1977). Prostaglandin and prostaglandin synthetase in the cricket, Acheta domesticus. J. Insect Physiol. 23, 905-911.

Dunn, P. E., and Drake, D. R. (1983). Fate of bacteria injected into naïve and immunized larvae of the tobacco hornworm Manduca sexta. J. Invertebr. Pathol. 41, 77-85.
Figueiredo, M. B., Genta, F. A., Garcia, E. S., and Azambuja, P. (2008). Lipid mediators and vector infection: Trypanosoma rangeli inhibits Rhodnius prolixus hemocyte phagocytosis by modulation of phospholipase A2 and PAF-acetylhydrolase activities. J. Insect Physiol. 54, 1528-1537.

Garcia, E. S., Machado, E. M. M., and Azambuja, P. (2004). Inhibition of hemocyte microaggregation reactions in Rhodnius prolixus larvae orally infected with Trypanosoma rangeli. Exp. Parasitol. 107, 31-38.

Goldsworthy, G., Mullen, L., OpokuWare, K., and Chandrakant, S. (2003). Interactions between the endocrine and immune systems in locusts. Physiol. Entomol. 28, 54-61.

Hatae, N., Sugimoto, Y., and Ichikawa, A. (2002). Prostaglandin receptors: advances in the study of EP3 receptor signaling. J. Biochem. 131, 781-784.

Jurenka, R. A., Pedibhotla, V. K., and Stanley, D. W. (1999). Prostaglandin production in response to a bacterial infection in true armyworm larvae. Arch. Insect Biochem. Physiol. 41, 225-232.

Kim, G. S., Nalini, M., Kim, Y., and Lee, D.-W. (2009). Octopamine and 5-hyddroxytryptamine mediate hemocytic phagocytosis and nodule formation via eicosanoids in the beet armyworm, Spodoptera exigua. Arch. Insect Biochem. Physiol. 70, 162-176.

Kim, Y., Ji, D., Cho, S., and Park, Y. (2005). Two groups of entomopathogenic bacteria, Photorhabdus and Xenorhabdus, share an inhibitory action against phospholipase A2 to induce host immunodepression. $J$. Invertebr. Pathol. 89, 258-264.

Kim, Y., Jung, S., and Madanagopal, N. (2008). Antagonistic effect of juvenile hormone on hemocytespreading behavior of Spodoptera exigua in response to an insect cytokine and its putative membrane action. J. Insect Physiol. 54, 909-915.

Lemaitre, B., and Hoffmann, J. (2007). The host defense of Drosophila melanogaster. Annu. Rev. Immunol. 25, 697-743.

Loher, W. (1979). The influence of prostaglandin E2 on oviposition in Teleogryllus commodus. Entomol. Exp. Appl. 25, 197-119.

Loher, W. (1984) "Behavioral and physiological changes in cricket-females after mating," in Advances in Invertebrate Reproduction, Vol. 3, ed. W. Engels (London: Elsevier), 189-201.

Loher, W., and Edson, K. (1973). The effect of mating on egg production and release in the cricket, Teleogryllus commodus. Entomol. Exp. Appl. 16, 483-490.

Loher, W., Ganjian, I., Kubo, I., StanleySamuelson, D. W., and Tobe, S. S. (1981). Prostaglandins: their role in egg-laying in the cricket Teleogryllus commodus. Proc. Natl. Acad. Sci. U.S.A. 78, 7835-7838.

Lord, J. C., Anderson, S., and Stanley, D. W. (2002). Eicosanoids mediate Manduca sexta cellular response to the fungal pathogen Beauveria bassiana: a role for the lipoxygenase pathway. Arch. Insect Biochem. Physiol. 51, 46-54.

Machado, E., Swevers, L., Sdralia, N., Medeiros, M. N., Mello, F. G., and Iatrou, K. (2007). Prostaglandin signaling and ovarian follicle development in the silkmoth, Bombyx mori. Insect Biochem. Mol. Biol. 37, 876-885.

Mandato, C. A., Diehl-Jones, W. L., Moore, S. J., and Downer, R. G. H. (1997). The effects of eicosanoid biosynthesis inhibitors on prophenoloxidase activation, phagocytosis and cell spreading in Galleria mellonella. J. Insect Physiol. 43, 1-8.

Marin, D., Dunphy, G. B., and Mandato, C. A. (2005). Cyclic AMP affecdts the hemocyte responses of larval Galleria mellonella to selected antigens. J. Insect Physiol. 51, 575-586.

Merchant, D., Ertl, R. L., Rennard, S. I., Stanley, D. W., and Miller, J. S. (2008). Eicosanoids mediate insect hemocyte migration. J. Insect Physiol. 54, 215-221.

Miller, J. S. (2005). Eicosanoids influence in vitro elongation of plasmatocytes from the tobacco hornworm, Manduca sexta. Arch. Insect Biochem. Physiol. 59, 42-51.

Miller, J. S., Nguyen, T., and Stanley-Samuelson, D. W. (1994). Eicosanoids mediate insect nodulation responses to bacterial infections. Proc. Natl. Acad. Sci. U.S.A. 91, 12418-12422.

Park, Y., and Kim, Y. (2000). Eicosanoids rescue Spodoptera exigua infected with Xenorhabdus nematophilus, the symbiotic bacteria to the entomopathogenic nematode Steinernema carpocapsae. J. Insect Physiol. 46, 1469-1476.

Park, Y., Kim, Y., Putnam, S. M., and Stanley, D. W. (2003). The bacterium Xenorhabdus nematophilus depresses nodulation reactions to infection by inhibiting eicosanoid biosynthesis in tobacco hornworm, Manduca sexta. Arch. Insect Biochem. Physiol. 52, 71-80.

Park, Y., Kim, Y., and Stanley, D. W. (2004). The bacterium Xenorhabdus nematophila inhibits phospholipases 
A2 from insect, prokaryote and vertebrate sources. Naturwissenschaften 91, 371-373.

Park, Y., and Stanley, D. W. (2006). The bacterium Xenorhabdus nematophilus impairs insect immunity by inhibition of eicosanoid biosynthesis in adult cricket, Gryllus firmus. Biol. Control 38, 247-253.

Petzel, D. H., Parrish, A. K., Ogg, C. L., Witters, N. A., Howard, R. H., and Stanley-Samuslson, D. W. (1993). Arachidonic acid and prostaglandin E2 in Malpighian tubules of yellow fever mosquitoes. Insect Biochem. Mol. Biol. 23, 431-437.

Petzel, D. H., and Stanley-Samuelson, D. W. (1992). Inhibition of eicosanoid biosynthesis modulates basal fluid secretion in the Malpighian tubules of the yellow fever mosquito (Aedes aegypti). J. Insect Physiol. 38, 1-8.

Phelps, P. K., Miller, J. S., and Stanley, D. W. (2003). Prostaglandins, not lipoxygenase products, mediate insect microaggregation reactions to bacterial challenge in isolated hemocyte preparations. Comp. Biochem. Physiol. 136, 409-416.

Qian, Y., Essenberg, R. C., Dillwith, J. W., Bowman, A. S., and Sauer, J. R. (1997). A specific prostaglandin E2 receptor and its role in modulating salivary secretion in the female tick, Amblyomma americanum (L.). Insect Biochem. Mol. Biol. 27, 387-395.

Qian, Y., Yuan, J., Essenberg, R. C., Bowman, A. S., Shook, A. S., Dillwith, J. W., and Sauer, J. R. (1998). Prostaglandin E2 in the salivary glands of the female tick, Amblyomma americanum (L.): calcium mobilization and exocytosis. Insect Biochem. Mol. Biol. 28, 221-228.

Radallah, D., Nogaro, M., and Fournier, B. (1995). Arachidonic acid and prostaglandin E2 stimulate phospholipase $\mathrm{C}$ activity in the rectum of the African locust, Locusta migratoria migratorioides. J. Insect Physiol. 41, 7-16.

Rolff, J., and Siva-Jothy, M. T. (2003). Invertebrate ecological immunology. Science 301, 472-475.

Schmid, M. R., Brockmann, A., Pirk, C. W. W., Stanley, D. W., and Tautz, J.
(2008). Adult honeybees (Apis mellifera L.) abandon hemocytic, but not phenoloxidase-based immunity. J. Insect Physiol. 54, 439-444.

Shrestha, S., and Kim, Y. (2007). An entomopathogenic bacterium, Xenorhabdus nematophila, inhibits hemocyte phagocytosis of Spodoptera exigua by inhibiting phospholipase A2. J. Invertebr. Pathol. 96, 64-70.

Shrestha, S., and Kim, Y. (2008). Eicosanoid mediates prophenoloxidase release from oenocytoids in the beet armyworm, Spodoptera exigua. Insect. Biochem. Mol. Biol.38, 99-112.

Shrestha, S., and Kim, Y. (2009). Oenocytoid cell lysis to release prophenoloxidase is induced by eicosanoid via protein kinase C. J. Asia Pac. Entomol. 12, 301-305.

Shrestha, S., and Kim, Y. (2010). Activation of immune-associated phsopholipase A2 is functionally linked to Toll/Imd pathways in the red flour beetle, Tribolium castaneum. Dev. Comp. Immunol. 34, 530-537.

Shrestha, S., Stanley, D., and Kim, Y. (2011). PGE2 induces oenocytoids cell lysis via a $G$ protein-coupled receptor in the beet armyworm, Spodoptera exigua. J. Insect Physiol. 57, 1568-1576.

Srikanth, K., Park, J., Stanley, D., and Kim, Y. (2011). Plasmatocyte spreading peptide influences hemocyte behavior via eicosanoids. Arch. Insect Biochem. Physiol. 78, 145-160.

Stanley, D. W. (2000). Eicosanoids in Invertebrate Signal Transduction Systems. Princeton: Princeton University Press.

Stanley, D. W. (2005). "Eicosanoids," in Comprehensive Insect Molecular Science, Vol. 4, eds L. I. Gilbert, K. Iatrou, and S. S. Gill (Amsterdam: Elsevier), 307-339.

Stanley, D. W. (2006). Prostaglandins and other eicosanoids in insects: biological significance. Annu. Rev. Entomol. 51, 25-44.

Stanley, D. W., Goodman, C., An, S., McIntosh, A., and Song, Q. (2008). Prostaglandins Al and E1 influence gene expression in an established insect cell line (BCIRLHzAM1 cells). Insect Biochem. Mol. Biol. 38, 275-282.

Stanley, D. W., Miller, J., and Tunaz, H. (2009). Eicosanoid actions in insect immunity. J. Innate Immun. 1 , 282-290.

Stanley-Samuelson, D. W., Jensen, E., Nickerson, K. W., Tiebel, K., Ogg, C. L., and Howard, R. W. (1991). Insect immune response to bacterial infection is mediated by eicosanoids. Proc. Natl. Acad. Sci. U.S.A. 88, 1064-1068.

Stanley-Samuelson, D. W., and Loher, W. (1983). Arachidonic and other long-chain polyunsaturated fatty acids in spermatophores and spermathecae of Teleogryllus commodus: significance in prostaglandinmediated reproductive behavior. $J$. Insect Physiol. 29, 41-45.

Stanley-Samuelson, D. W., Peloquin, J. J., and Loher, W. (1986). Egg-laying in response to prostaglandin injections in the Australian field cricket, Teleogryllus commodus. Physiol. Entomol. 11, 213-219.

Stroschein-Stevenson, S. L., Foley, E. O'Farrell, P. H., and Johnson, A. D. (2006). Identification of Drosophila gene products required for phagocytosis of Candida albicans. PLoS Biol. 4, 87-99. doi:10.1371/journal.pbio.0040004

Tunaz, H., Park, Y., Buyukguzel, K., Bedick, J. C., Nor Aliza, A. R., and Stanley, D. W. (2003). Eicosanoids in insect immunity: bacterial infection stimulates hemocytic phospholipase A2 activity in tobacco hornworms. Arch. Insect Biochem. Physiol. $52,1-6$

Van Kerkhove, E., Pirotte, P., Petzel, D. H., and Stanley-Samuelson, D. W. (1995). Eicosanoid biosynthesis inhibitors modulate basal fluid secretions rates in the Malpighian tubules of the ant, Formica polyctena. J. Insect Physiol. 41, 435-441.

Vanden Broeck, J. (2001). Insect $\mathrm{G}$ protein-coupled receptors and signal transduction. Arch. Insect Biochem. Physiol. 48, 1-12.

von Euler, U. S. (1936). On the specific vasodilating and plain muscle stimulating substances from accessory genital glands in men and certain animals (prostaglandin and vesiglandin). J. Physiol. 88, 213-234.

Wagner, C., Isermann, K., Fehrenbach, H., and Roeder, T. (2008). Molecular architecture of the fruit fly's airway epithelial immune system. BMC Genomics 9, 446. doi:10.1186/1471-2164-9-446

Weinheimer, A. J., and Spraggins, R. L. (1969). The occurrence of two new prostaglandin derivatives (15epi-PGA2 and its acetate, methyl ester) in the Gorgonian, Plexaura homomalla. Chemistry of coelenterates. XV. Tetrahedron Lett. 59, 5185-5188.

Yajima, M., Takada, M., Takahashi, N., Kikuchi, H., Natori, S., Oshima, Y. and Kurata, S. (2003). A newly established in vitro culture using transgenic Drosophila reveals functional coupling between the phospholipase A2-generated fatty acid cascade and lipopolysaccharide-dependent activation of the immune deficiency (imd) pathway in insect immunity. Biochem. J. 371, 205-210.

Conflict of Interest Statement: The authors declare that the research was conducted in the absence of any commercial or financial relationships that could be construed as a potential conflict of interest.

Received: 31 October 2011; paper pending published: 01 December 2011; accepted: 05 December 2011; published online: 30 December 2011.

Citation: Stanley D and Kim Y (2011) Prostaglandins and their receptors in insect biology. Front. Endocrin. 2:105. doi: 10.3389/fendo.2011.00105

This article was submitted to Frontiers in Experimental Endocrinology, a specialty of Frontiers in Endocrinology.

Copyright () 2011 Stanley and Kim. This is an open-access article distributed under the terms of the Creative Commons Attribution Non Commercial License, which permits non-commercial use, distribution, and reproduction in other forums, provided the original authors and source are credited. 